Polish Journal of Microbiology

2011, Vol. 60, No 2, 95-103

MINIREVIEW

\title{
Staphylococcal Cassette Chromosome mec (SCCmec) Classification and Typing Methods: an Overview
}

\author{
AGATA TURLEJ ${ }^{1}$, WALERIA HRYNIEWICZ ${ }^{1}$ and JOANNA EMPEL ${ }^{2}$
}

${ }^{1}$ Department of Epidemiology and Clinical Microbiology, ${ }^{2}$ Department of Molecular Microbiology National Medicines Institute, Warsaw, Poland

Received 6 April 2011, accepted 6 May 2011

Abstract

Meticillin-resistant Staphylococcus aureus (MRSA) is one of the main causes of hospital-acquired infections, but since late 1990s also the community-acquired. For better understanding of the S. aureus epidemiology there is an urgent need for creation of new typing method for SCCmec element. The molecular typing of MRSA for epidemiological purposes is investigated by pulsed-field gel electrophoresis (PFGE), multilocus sequence typing (MLST), spa typing and the SCCmec type assignment. In last few years not only new type of SCCmec (VI to XI) have been identified, but also additional subtypes (i.e. IVg-j) and different variants of already existed one (i.e. 5C2\&5 and 2B2\&5) were discovered. The aim of this review is to briefly summarize current knowledge about SCCmec classification and to discuss advantages and disadvantages of selected SCCmec typing methods.

Ke y word s: Staphylococcus aureus (MRSA), SCCmec classification and typing

\section{Introduction}

Staphylococcus aureus is one of the leading causes of bacterial infections in developed countries and is responsible for a wide spectrum of diseases, ranging from minor skin infections to fatal necrotizing pneumonia. Since the introduction of penicillin into medical treatment in early 1940s, the resistance for beta-lactams has started to develop. It was a result of the acquisition of a plasmid, coding for penicillinase, a penicillin-hydrolyzing enzyme, which is able to cleave the beta-lactam ring and thus inactivate antibiotic molecule. Penicillin resistant strains soon spread not only in healthcare facilities, but also in the community. To overcome infections caused by beta-lactamase-producing S.aureus, a narrow spectrum semi-synthetic penicillin (meticillin) was introduced. However, soon after that, in 1961, first meticillin-resistant Staphylococcus aureus (MRSA) strain was identified. Initially, MRSA strains were encountered only in the hospitals, but in the late 1990s first virulent community-acquired MRSA (CA-MRSA) clones, characterized by the presence of the toxin Panton-Valentine leukocidin (PVL), appeared rapidly and unexpectedly. They quickly spread worldwide, initially only in the community, but later on also in the healthcare facilities, displacing in some caun- tries typical HA-MRSA. For this reason, nowadays, distinction between CA-MRSA and mostly multiresistant HA-MRSA become challenging (Chambers and Deleo, 2009; Deurenberg and Stobberingh, 2008). The resistance of $S$. aureus to meticillin is caused by the presence of the mecA gene, encoding for an additional $78-\mathrm{kD}$ a penicillin binding protein $2 \mathrm{a}$, (PBP2a or PBP2'). Compared to other PBP, PBP2a has a low affinity for all beta-lactam antibiotics. As a result of that, even in the presence of a beta-lactam antibiotic, the peptidoglycan layer biosynthesis is not disrupted and the bacterium can survive (Berger-Bachi and Rohrer, 2002; Deurenberg and Stobberingh, 2008). The mecA gene is located within a mec operon together with its regulatory genes: $m e c I$ and mecR1. (Berger-Bachi and Rohrer, 2002). The mec operon is carried by staphylococcal cassette chromosome mec (SCCmec). The origin of SCCmec is still unknown, but it is proposed that it was acquired by $S$. aureus from S. sciuri and that the mecA-positive coagulase-negative staphylococci (CoNS), especially S. epidermidis, may be a potential reservoir for the SCCmec element (Mongkolrattanothai et al., 2004; Wu et al., 2001). On the other hand, it is suggested that the main source of SCCmec could be MRSA itself (Aires de Sousa and de Lencastre, 2004). There are also suggestions of possible acquisition of the mecA

\footnotetext{
* Corresponding author: Agata Turlej, National Medicine Institute, Chełmska 30/34, 00-725 Warsaw, Poland; phone: (48-22) 841 57 69; fax: (48-22) 8412949; e-mail: aturlej@cls.edu.pl
} 
region from $S$. fleurettii, which is a commensal bacterium of animals (Tsubakishita et al., 2010). SCCmec typing, which classifies SCCmec elements on the basis of their structural differences, is applied in epidemiological studies to distinguish MRSA strains or to define an MRSA clone in combination with the genotype of meticillin-susceptible S. aureus (MSSA) strain in which a SCCmec element has integrated.

\section{SCCmec element composition}

SCCmec elements, detected in almost all MRSA strains, belong to particular type of the staphylococcal mobile genetic elements coding for meticillin-resistance and designated as staphylococcal cassette chromosome mec (Katayama et al., 2000). In S. aureus strains, SCCmec elements always integrate sequence specifically at the unique site of the chromosome, attBscc (bacterial chromosomal attachment site). The attBscc is located near the origin of replication, at the 3' end of $\operatorname{orf} X$, coding for an open reading frame X of unknown function, well conserved among both MRSA and MSSA strains (Hiramatsu et al., 2001; Ito et al., 1999; Ito et al., 2001). The attachment site contains a core 15-bp sequence, called the integration site sequence (ISS), that is necessary for $c c r$-mediated recombination (IWG-SCC, 2009; Katayama et al., 2000). After integration of SCCmec into the chromosome, ISS is found in direct repeat sequences at left and right SCCmec/chromosomal junctions of the integrated SCCmec element. Different SCCmec elements share similar backbone structure, that consists of (i) mec complex, composed of mecA operon, (ii) $c c r$ gene complex, composed of cassette chromosome recombinase (ccr) gene(s) and (iii) three regions bordering the $c c r$ and mec complexes, designated as joining $(\mathrm{J})$ regions. The composition of almost all SCCmec elements identified so far in $S$. aureus can be presented as follow: (orfX)J3-mec-J2-ccr-J1 (Chongtrakool et al., 2006; Hiramatsu et al., 2002). The exception constitute SCCmecVII and a newly described SCCmecIX, with the ccr gene complex positioned between $\mathrm{J} 3$ and $\mathrm{J} 2$ regions and the mec gene complex between $\mathrm{J} 2$ and $\mathrm{J} 1$ regions (Berglund et al., 2008; Li et al., 2011). It is noteworthy, that different authors, and sometimes even the same, present the structure of the same SCCmec elements in reverse orientations, what creates difficulties or even confusions, especially for these not experienced in the field. The orientation with SCCmec located at the right site of $\operatorname{orfX}$ gene seems to be more correct as it is consistent with direction on genomic maps of $S$. aureus and will be used for needs of this article. Since the structural components mentioned above play a crucial role in classification of SCCmec elements they will be presented below in more details.
The ccr gene complex. The ccr gene complex is composed of the $c c r$ gene(s) surrounded by orfs. The $c c r$ genes encode for DNA recombinases of the invertaseresolvase family, enzymes that can catalyze precise excision of the SCCmec as well as its integration, both site- and orientation-specific, into staphylococcal chromosome, being thus responsible for mobilization of the cassette (Katayama et al., 2000). Based on the composition of $c c r$ genes, two distinct $c c r$ gene complexes have been reported to date, one carrying two adjacent $c c r$ genes, $c c r A$ and $c c r B$, and the second carrying $c c r C$. The $c c r A$ and $c c r B$ genes identified to date in $S$. aureus strains have been classified into four and five allotypes respectively, resulting in six $c c r$ gene complex types, designated as type 1 (ccrA1B1), type 2 (ccrA2B2), type 3 (ccrA3B3), type 4 (ccrA4B4), type 7 (ccrA1B6) and type 8 ( $c c r A 1 B 3)$. All identified so far $c c r C$ variants have shown high nucleotide similarity and are assigned to only one allotype, $c \mathrm{crC1}$, constituting type 5 of $c \mathrm{cr}$ gene complex (Chongtrakool et al., 2006; IWG-SCC, 2009) (http://www.sccmec.org/).

The mec gene complex. Two evolutionary different lineages of mec gene complexes have been described in S. aureus. The first one, which encompasses the vast majority of known and well characterized mec gene complexes, have been observed in MRSA isolates of human origin since the nineties. The prototype of this lineage is the mec gene complex designated as class A, composed of an intact mec operon, the hyper-variable region (HVR) and the insertion sequence IS431 (Ito et al., 2001; Katayama et al., 2001). The mec operon includes mecA gene and located upstream of mecA its regulatory genes: $m e c R 1$ and $m e c I$, coding for the signal transducer and the repressor, respectively. Differences between class A mec gene complex and other mec gene complexes of this lineage, described to date, result mainly from insertions of IS elements, IS 1272 or IS431, into the region of $m e c A$ regulatory genes, causing complete deletion of $m e c I$ and, different in size, partial deletions of $m e c R 1$. Depending on the structural diversity of mecI-mecR1 region, five major classes of mec gene complexes, of the said lineage, have been defined by IGW-SCC (IWG-SCC, 2009):

- Class A, which contains intact mec gene complex: IS431-mecA-mecR1-mecI;

- Class B, where mecR1 is truncated by insertion sequence IS1272: IS431-mecA- $\Delta m e c R 1-I S 1272$;

- Class C1, where mecR1 is truncated by insertion sequence IS431 having the same direction as the IS431 downstream of mecA: IS431-mecA- $\Delta m e c R 1-$ -IS431;

- Class C2, where mecR1 is truncated by insertion sequence IS431 having the reverse direction to the IS431 downstream of mecA: IS431-mecA$\Delta m e c R 1-I S 431 ;$ and 
Table I

Additional resistance genes located on mobile elements within the SCCmec elements

\begin{tabular}{|c|c|c|c|}
\hline Genetic element & SCCmec type/subtype & Gene & Resistance \\
\hline \multirow[t]{2}{*}{ pUB110 } & \multirow[t]{2}{*}{ I, II, IVA } & ble & bleomycin \\
\hline & & ant4' & tobramycin \\
\hline \multirow[t]{2}{*}{$\operatorname{Tn} 554$} & \multirow[t]{2}{*}{ II, SCCHg,VIII } & ermA & erythromycin \\
\hline & & aad $9 / s p c$ & streptomycin / spectinomycin \\
\hline $\mathrm{SCC}_{\mathrm{Hg}}$ & - & mer & mercury \\
\hline pT181 & III & tet & tetracycline \\
\hline$\Psi \operatorname{Tn} 554$ & III & cad & cadmium \\
\hline $\operatorname{Tn} 4001$ & IV (IVc and 2B\&5) & $a a c \mathrm{~A}-a p h \mathrm{D}$ & aminoglycosides \\
\hline
\end{tabular}

- Class D, where mecR1 is partly deleted but there is no IS element downstream of $\Delta m e c R 1$ : IS431$m e c A-\Delta m e c R 1$. This class has been observed in S. caprae only (Katayama et al., 2001).

Recently, data from genome sequencing project of the bovine $S$. aureus isolate LGA251, have revealed a mec gene complex of the second evolutionary lineage (McCarthy and Lindsay, 2010)) (http://www.sanger. ac.uk/pathogens). This new complex, depicted as: bla $\mathrm{Z}$-mec $A_{\mathrm{LGA} 251}-m e c R 1_{\mathrm{LGA251}}-m e c I_{\mathrm{LGA} 251}$, constitutes the sixth defined major class, assigned as class $\mathrm{E}$ (http:// www.sccmec.org/).

Besides the major classes of the mec gene complex several variants within the classes have also been distinguished, for example: class $\mathrm{A}_{3}$, where $m e c I$ is truncated by insertion sequence IS1182: IS431-mecA-mecR1$\Delta m e c I$-IS1182 and class $\mathrm{A}_{4}$, where mecI is disrupted by insertion sequence IS1182: IS431-mecA-mecR1- $\Delta$ mecIIS1182- $\Delta$ mecI (Shore et al., 2005) or class $B_{2}$, which has an insertion of the transposone $\operatorname{Tn} 4001$ upstream of $m e c A$ in $\Delta m e c R 1$ : IS431-mecA- $\Delta m e c R 1-$ Tn4001-IS1272 (Heusser et al., 2007).

The joining $(\mathrm{J})$ regions. Apart from the $\mathrm{ccr}$ and $m e c A$ gene complexes, essential for the SCCmec biological functions, the cassette comprises also three joining regions (J1-J3), previously called "junkyard" regions (Hiramatsu etal., 2002). J regions from different SCCmec elements are arranged in the same order. The $\mathrm{J} 1$ region is located at right site of the cassette, the $\mathrm{J} 2$ region between the $c \mathrm{cr}$ and the mec complexes and the $\mathrm{J} 3$ region at the left chromosomal junction adjacent to orfX (IWG-SCC, 2009). Although considered as less important in terms of SCCmec functions, these regions are epidemiologically significant since they may serve as targets for plasmids or transposons, carrying additional antibiotic and heavy metal resistance determinants. Acquisition and accumulation of resistance genes by mobile elements like SCCmec enables their dissemination and in consequence leads to emerge of multidrug resistance strains. Examples of antibiotic resistance determinants, that may be carried within
J regions are summarized in Table I (Ito et al., 2003; Malachowa and DeLeo, 2010). Sequence analysis of $\mathrm{J}$ regions from different SCCmec elements revealed that they are unique to particular types of $c \mathrm{cr}$-mec gene complex combinations and that variations of these regions within the same ccr-mec gene complex combination are specific for SCCmec subtypes (Hisata et al., 2005; Ito et al., 2003; IWG-SCC, 2009; Kwon et al., 2005; Ma et al., 2002; Ma et al., 2006; Milheirico et al., 2007b; Oliveira et al., 2001; Oliveira and de Lencastre, 2002; Shore et al., 2005).

\section{The SCCmec element classification}

The first SCCmec element was identified in Japanese S. aureus strain, N315 in 1999 and shortly after two additional SCCmec from different MRSA strains were determined (Ito et al., 1999; Ito et al., 2001). Based on detailed structural analysis these three SCCmec elements were classified as types I to III (Ito et al., 2001). In time, both new types of SCCmec, such as SCCmecIV (Ma et al., 2002), SCCmecV (Ito et al., 2004), SCCmecVI (Oliveira et al., 2006a), SCCmecVII (Berglund et al., 2008), SCCmecVIII (Zhang et al., 2009), SCCmecIX, SCCmecX (McCarthy and Lindsay, 2010), SCCmecXI (http://www.sccmec.org/) and many new variants of already known SCCmec types have been reported (BoyleVavra et al., 2005; Cha et al., 2005; Chlebowicz et al., 2010; Heusser et al., 2007; Higuchi et al., 2008; Hisata etal., 2005; Kwon etal., 2005; O’Brien etal., 2005; Oliveira and de Lencastre, 2002; Shore et al., 2005; Shukla et al., 2004; Stephens et al., 2007; Takano et al., 2008). With growing number of SCCmec types, subtypes or variants published in the literature it became obvious that without approved international rules of nomenclature system it would be difficult in the nearest future to keep in order suitable naming of new emerged SCCmec elements. To meet the urgent need the International Working Group on Classification of Staphylococcal Cassette Chromosome (SCC) Elements 
Table II

Reference strains for SCCmec types, which have been described up to date

\begin{tabular}{|l|l|l|l|l|l|}
\hline \multicolumn{1}{|c|}{$\begin{array}{c}\text { SCCmec } \\
\text { type/subtype }\end{array}$} & $\begin{array}{c}\text { GenBank } \\
\text { accession no }\end{array}$ & \multicolumn{1}{|c|}{ Origin } & \multicolumn{1}{|c|}{$\begin{array}{c}\text { Isolation } \\
\text { date }\end{array}$} & \multicolumn{1}{|c|}{ Description } \\
\hline I (1B) & NCTC 10442 & AB033763 & UK & 1961 & (Ito et al., 2001) \\
\hline II (2A) & N315 & D86934 & Japan & 1982 & (Ito et al., 1999) \\
\hline III (3A) & $85 / 2082$ & AB037671 & New Zealand & 1985 & (Ito et al., 2001) \\
\hline IVa (2B) & CA05 & AB063172 & USA & 1999 & (Ma et al., 2002) \\
\hline IVb (2B) & $8 / 6-3 P(J C S C 1978)$ & AB063173 & USA & 1996 & (Ma et al., 2002) \\
\hline IVc (2B) & $81 / 108(M R 108)$ & AB096217 & Japan & NA ${ }^{*}$ & (Ito et al., 2003) \\
\hline IVd (2B) & JCSC4469 & AB097677 & Japan & 1982 & (Ma et al., 2006) \\
\hline IVg (2B) & M03-68 & DQ106887 & Korea & 2003 & (Kwon et al., 2005) \\
\hline IVh (2B) & HAR22 & NA & Finland & 2002 & (Milheirico et al., 2007b) \\
\hline IVi (2B) & JCSC6668 & AB425823 & Sweden & 1999 & (Berglund et al., 2009) \\
\hline IVj (2B) & JCSC6670 & AB425824 & Sweden & 1990 & (Berglund et al., 2009) \\
\hline V (5C2) & WIS (JCSC3624) & AB121219 & Australia & 1999 & (Ito et al., 2004) \\
\hline VI (4B) & HDE 288 & AF411935 & Portugal & 1996 & (Oliveira et al., 2006a) \\
\hline VII (5C1) & JCSC6082 & AB373032 & Sweden & 2002 & (Berglund et al., 2008) \\
\hline VIII (4A) & C10682 & FJ670542 & Canada & 2003 & (Zhang et al., 2009) \\
\hline IX 1(C2) & JCSC6943 & NA & Thailand & 2006 & (Li et al., 2011) \\
\hline X (7C1) & JCSC6945 & NA & Canada & 2006 & (Li et al., 2011) \\
\hline XI (8E) & LGA251 & Na & NA & http://www.sccmec.org/ \\
\hline
\end{tabular}

* NA - information not available

(IWG-SCC) was established in 2009. The main objectives of the group was to define consensus rules of a uniform nomenclature system for SCCmec elements, determine minimum requirements for the description of the new SCCmec elements and establish guidelines for the identification of SCCmec elements for epidemiological studies (IWG-SCC, 2009). In published guidelines IWG-SCC decided to retain the previous nomenclature of SCCmec with additional information about combination of $c c r$ complex type and class of mec complex present in the element. Thus, classification of SCCmec element into the types (SCCmec typing), should be based on the combination of the type of $\mathrm{ccr}$ gene complex and the class of the mec gene complex present in the cassette, while variants within SCCmec types (SCCmec subtyping) should be defined by differences in their J regions, as it was proposed earlier by Hiramatsu group (Chongtrakool et al., 2006; IWG-SCC, 2009). Accordingly, SCCmec type I, was described additionally as $1 \mathrm{~B}$, what indicates the SCCmec element harboring the type $1 \mathrm{ccr}$ and a class B mec gene complexes. To date, eleven SCCmec types have been defined. The other known SCCmec types are designated type II (2A), type III (3A), type IV (2B), type V (5C2), type VI (4B), type VII (5C1), type VIII (4A), type IX (1C2), type X (7C1) and type XI (8E). They all are summarized in Table II.

The improved way of SCCmec classification allowed also to assign the mosaic variants of SCCmec. For example, SCCmec element from ZH47 strain, harboring type $2 \mathrm{ccr}$ gene complex and additional $c c r C 1$ in combination with mec class $\mathrm{B}_{2}$ was designated type IV (2B\&5), while SCCmec element from TSGH17 and PM1 strains, carrying two different $c c r C 1$ allels, 2 and 8, that was previously reported as SCCmec type VII or Taiwanese SCCmec type V $\left(\mathrm{SCCmecV}_{\mathrm{T}}\right)$, was designated type V (5C2\&5) (Boyle-Vavra et al., 2005; Heusser et al., 2007; Higuchi et al., 2008; IWG-SCC, 2009; Takano et al., 2008).

Due to the increasing diversity among SCCmec subtypes, IWG-SCC proposed the preparation of a computerized system, which will be able to characterize and assign certain SCCmec subtype based on the occurrence of specific elements within the J regions.

Together with the discovery of new SCCmec types, also a need for new more complex SCCmec typing methods has emerged.

\section{Available SCCmec typing methods}

It had already been observed that the worldwide spread of MRSA is driven by the dissemination of a number of clones with a specific genetic background. Epidemiological studies revealed that for proper clone assignment not only the multilocus sequence typing (MLST) and spa typing is required, but also SCCmec typing is needed (Deurenberg et al., 2007). Since that 
time, it has become necessary to find an easy and robust method for SCCmec element identification and typing. The more complex our knowledge about SCCmec elements is, the more challenging invention of the typing method becomes and the more relevant becomes the question, how accurate should we be in assigning SCCmec element type for epidemiological purposes. The SCCmec typing methods has been developed along with the new SCCmec types descriptions and appearance of the novel techniques or approaches for their analysis. Three different schemes of SCCmec typing can be distinguished: methods based on the restriction enzymes digestion, methods based on PCR or multiplex PCR (M-PCR) and methods based on real-time PCR.

First SCCmec typing methods. The first methods for detecting polymorphisms in the mecA vicinity were based on hybridizations of the mecA probe and Tn554 probe with ClaI-digested genomic DNA from the analyzed isolates (Leski et al., 1998). This method was very useful for epidemiological studies before the SCCmec element's structure was described. Nowadays there are some concepts on using restriction enzymes digestion in combination with PCR, like in multienzyme multiplex PCR-amplified fragment length polymorphism (ME-AFLP) or in SCCmec typing method by PCR amplification of the $c c r B$ gene in combination with restriction fragment length polymorphism (RFLP) employing endonucleases HinfI and BsmI (van der Zee et al., 2005; Yang et al., 2006).

In the first one, the obtained typing patterns were found to cluster together according to the SCCmec type of the strain, with the discriminatory power comparable to PFGE. However, it is just a pattern based typing, which might be an interesting method for prescreening of a large strain collection, but is by far not sensitive enough to proper assign SCCmec type for epidemiological purposes, since it does not recognize any characteristic features for already described SCCmec elements. The second method is simple and time-effective, but far not elaborate enough, because it focuses only on the $\operatorname{cr} B$ typing. Since the $\operatorname{crr} C$ genes were also described, it is not complex enough to be useful, concerning the current knowledge of SCCmec types. Moreover, for proper SCCmec assignment the mec class description in combination with $c c r$ type is necessary. It seems that the scheme of SCCmec typing based on restriction enzymes digestion is no longer superior. The most common methods used nowadays for SCCmec typing based on PCR are summarized below.

PCR based SCCmec typing methods. During the past several years, a number of SCCmec typing methods based on multiplex PCR (M-PCR) have been developed (Boye et al., 2007; Hisata et al., 2005; Kondo et al., 2007; Milheirico et al., 2007a; Oliveira and de Lencastre, 2002; Zhang et al., 2005). Two different approaches were applied in this methods; one was focused on analysis of J regions, whereas the other determine mainly mec class and $c c r$ type. The first M-PCR method was described by Oliveira et al. (Oliveira and de Lencastre, 2002). At that time it was innovative technique that enabled to increase analysis scale and exchange the information about SCCmec types all over the world. It was based on identification of specific genes or motifs located mostly in the J regions of particular cassettes. Potentially, this method should detected SCCmec type I-IV, but in practice detection of SCCmec type III was problematic as in fact the primers were designed not to SCCmec type III but to so-called SCCmercury, which at that time was believed to be the integral part of the element. For more details see Chongtrakool et al. (Chongtrakool et al., 2006). This SCCmec typing strategy also did not discriminate SCCmec type IV and VI. (Oliveira et al., 2006a). In 2007, the same group published an update for Oliveira's method, which improved the detection of SCCmec type I to IV and includes the structure determination of the SCCmec type V and VI. However, the SCCmecVI was suggested to be confirmed by ccrB sequencing, which is costly and time-consuming (Milheirico et al., 2007a; Oliveira et al., 2006b) (http:// www.ccrbtyping.net). The most significant advantage of this method is that it is a quick, single-tube M-PCR reaction for all detectable by this method SCCmec types. Unfortunately, the method is still based on markers located within the J regions. This can cause some problems, as for example in our practice we sometimes see the pattern for SCCmec type I similar to type VI, which is confusing. Almost at the same time Zhang et al. has described a complex method for SCCmec I to V typing and SCCmec type IV subtyping in a multiplex PCR reactions (Zhang et al., 2005). This method include three M-PCR reactions and one single target PCR reaction with sets of primers specific to $m e c, c c r$ and J1 region. First M-PCR reaction uses a set of primers specific for SCCmec type I to V, with SCCmec subtypes IVa to IVd, the second M-PCR reaction uses primers for assigning mec class $\mathrm{A}$ and $\mathrm{B}$ and third M-PCR reaction uses primers for type 1-3 ccr described previously by Ito et al. For detection of type $5 \mathrm{ccr}$ the authors propose PCR reaction with single pair of primers (Ito et al., 2001; Zhang et al., 2005). The proposed approach seems to be not useful for large-scale analysis, since four separate reactions should be done. Moreover, it does not allow to detect neither mec class $\mathrm{C} 1$ and C2 nor type $4 \mathrm{ccr}$. On the other hand, this method allows to detect Taiwanese SCCmec type V (5C2\&5). This mosaic variant of SCCmec type $\mathrm{V}$ gives one extra band of $1599 \mathrm{bp}$, compared to the reference type $\mathrm{V}$ (5C2). Another SCCmec typing method was developed in 2005 by Hisata et al. who proposed another set of primers (Hisata et al., 2005). The method allowed 
to detect SCCmec type I, IIa, IIb, III and IVa to IVd and was based mainly on mec class and ccr type assignment. Unfortunately, it was not possible to perform the analysis in a single M-PCR reaction and the method did not become popular widely. Another recently developed technique was presented by Boye et al. (Boye et al., 2007). It is a quick and easy to interpret method based on single-tube M-PCR reaction, using primers for specific detection of both mec class and ccr type of SCCmec type I to V. It seems to be very useful for first screening of large amount of strains, but for the detection of SCCmec type I to III it is not complex enough. For example, to detect SCCmec type III only ccrC specific pair of primers is used, which confirms just the presence of SCCmercury (Boye et al., 2007). Moreover, this method also misclassifies SCCmec type I with type VI. However, this method can also be used for confirmation of doubtful SCCmec types. The most complex and promising system for SCCmec assignment, especially in the light of the new guidelines for SCCmec elements classification was developed by Kondo et al. (Kondo et al., 2007). This PCR scheme combines six M-PCR reactions: M-PCR 1 for amplification of $c c r$ type (1-4) along with mecA gene; M-PCR 2 for amplification of mec class (A, B and C2); M-PCR 3 for amplification of ORFs from J1 region of SCCmec type I and IV; M-PCR 4 for amplification of ORFs from J1 region of SCCmec type II, III and V; M-PCR 5 and 6 for amplification of gene alleles located in $\mathrm{J} 2$ and $\mathrm{J} 3$ region of SCCmec elements, respectively. M-PCRs 5 and 6 are used for the identification of integrated copies of transposons (Tn554 or $\Psi$ Tn554) and plasmids (pUB110 or pT181). The most significant advantage of this method is its flexibility, since it does not detect any particular SCCmec type, but only crucial loci, which in combination gives at the end the SCCmec type. This approach potentially allows detection of SCCmec types I to IX except SCCmecVII and X since primers specific for mec class $\mathrm{C} 1$ have not been included to this system yet. Using M-PCRs 3 to 6 it is possible also to identify variety of $\mathrm{SCC} m e c$ subtypes. However, since this method requires a relatively large number of $\mathrm{PCR}$ reactions to determine the structure of SCCmec it is quite complicated and time consuming. That is why it is suggested to perform just M-PCR 1 and 2 to assign type of SCCmec elements and in most cases, it may be enough for epidemiological purposes. Recently new SCCmec type V variants, which are getting epidemiologically important, were also described (Chlebowicz et al., 2010; Higuchi et al., 2008). It turned out that despite the high similarity among $\operatorname{ccrC} 1$ genes, its' specific alleles 1, 2, 8, 9 and 10 typing could be also important for SCCmec type V precise characterization. For detection of mosaic $\mathrm{SCCmecV}$ (5C2\&5) variant, Higuchi et al. provides a set of primers specific for two types $5 \mathrm{ccr}$ ( $\mathrm{ccrC} 1$ allel 2 and
ccrC1 allel 8), two characteristic ORFs (orf33 and orf35) and the mec class $\mathrm{C} 2$ variant, in which nucleotide substitution in IS431 results in truncated transposase in IIS431 in mec complex C2 (Higuchi et al., 2008). The last method we would like to mention here concerns detection of recently discovered SCCmec type VIII (McClure et al., 2010; Zhang et al., 2005). It is based on single-tube M-PCR reaction using a set of primers specific for detection of characteristic features of this particular of SCCmec element, namely type $4 \mathrm{ccr}$, mec class $\mathrm{A}$, and a unique junction within the $\mathrm{J}$ region as well as internal controls. Regarding the growing number of MRSA clones harbouring different SCCmec IV subtypes one of the most important issue become also invention of a robust method for SCCmec type IV subtyping. In our opinion dedicated to solve this problem is method published by Milheirico et al., which is to our knowledge the most complex among available methods and allow to detect SCCmec type IV subtypes from a to h (Milheirico et al., 2007b). We also use subtypig descriebed by Zhang et al., but we find that quite often it is difficult to detect SCCmec type IVc using this method, while we never had such problems when using Milheirico et al. method.The PCR based methods for SCCmec typing are the most common and the easiest to implement in laboratories, since they do not require additional expensive equipment, but on the other hand, they are labor and time-consuming, compared to realtime PCR based analysis.

Real-time PCR based SCCmec typing methods. In parallel to PCR based methods for SCCmec typing, the methods based on real-time PCR have also been developed. A multiplex scheme based on a real-time PCR targeting the $c \mathrm{crB}$ regions of SCCmec types I to IV was published in 2004 by Francois et al., but with current knowledge about SCCmec types this method is not elaborate enough (Francois et al., 2004). Recently, however, a new approach using $c c r$-specific padlock probes and tag microarray analysis for simultaneous probing of core genome diversity and identification of SCCmec was developed. However, the set of padlock probes includes only oligonucleotides targeting diagnostic regions in the mecA, $c c r B$ and $c c r C$ genes without mec class recognition (Kurt et al., 2009). A significant disadvantage of these methods is that they detect only $c c r$ locus and ignore the mec complex, which may result in misclassification and do not allow the detection of novel combinations of the mec and ccr complex in SCCmec elements. On the other hand, in the same year, a very interesting method for SCCmec element typing was published, based on a rapid molecular beacon real-time PCR (MB-PCR) assay (Chen et al., 2009). The design of the system is based on the definition of SCCmec types as a combination of the ccr allotype along with the mec class complex. The assay con- 
sists of two multiplex panels, the combination of which results in two targets ( $m e c$ class, $c c r$ ) for each SCCmec type. MB-PCR panel I targets mecA, ccrB2, mecI, and the $\Delta m e c R 1-I S 1272$ junction (mec class B) and thus can definitively identify SCCmec types II and IV. MB-PCR panel II detects $c c r C, c c r B 1, c c r B 3, c c r B 4$, and the $\Delta m e c$ R1-IS431 junction ( $m e c$ class $\mathrm{C} 2$ ) and is therefore capable of identifying SCCmec types I, III, V, and VI in combination with panel I. This method can also detect the recently described novel SCCmec type VIII (ccrAB4 with mec class A). The authors of this method ascertain that it is possible to easily classify isolates within 3 to $4 \mathrm{~h}$, including DNA isolation, PCR cycling and analysis, which is extremely quick. However, in this analysis it is impossible to detect SCCmec subtypes, since there are no primers designed for J-regions and this method does not detect the mec class $\mathrm{C} 1$, which is characteristic for SCCmec type VII and X.

The most significant advantage of real-time PCR based methods is the small amount of time and labor required for the analysis. They do not combine many preparatory steps and are easy to interpret. On the other hand, they require special equipment and reagents, which are very expensive.

\section{Conclusion}

A variety of different methods for SCCmec typing are now available. To cope with the increasing diversity of SCCmec elements being reported, methods for their detection should be elaborated and flexible. Conventional PCR assays using commonly up to ten primer pairs in a single-tube assay can give various sensitivities, depending on the template quality and may be easily contaminated. On the other hand, the real-time PCR assay requires expensive reagents and instruments, which can limit its use in many microbiological laboratories. Unfortunately, there is still no method available for SCCmec type VII and X-XI typing. High heterogenicity and variability of SCCmec elements make them complicated as a markers for epidemiological clone assignment. In our opinion, up to date, the best way of assigning the SCCmec type is to prepare the M-PCR 1 and 2 according to Kondo et al. or real-time PCR according to Chen et al., for ccr type and mec class detection. For SCCmec type IV subtyping we recommend method by Milheirico et al. (Milheirico et al., 2007b). This proceeding should be enough in most cases. For more accurate subtyping we suggest using the other M-PCRs described by Kondo et al. and if necessary also methods established by Higuchi et al. and McClure et al. Recent work done by IWG-SCC clarified the classification of the major SCCmec elements type, but there is still a lot of ambiguity regarding naming of the
SCCmec variants, which requires further investigations and agreements. This includes answering the question, how accurate should we be in assigning SCCmec element type/variant for epidemiological purposes.

\section{Acknowledgements}

This work was a part of the activities of the CONCORD Collaborative project supported by the European Commission grant HEALTH-F3-2008-222718. WH and JE was also partly supported by finding from the European Community MOSAR network contract LSHP-CT-2007-037941.

\section{Literature}

Aires de Sousa M. and H. de Lencastre. 2004. Bridges from hospitals to the laboratory: genetic portraits of methicillin-resistant Staphylococcus aureus clones. FEMS Immunol. Med. Microbiol. 40: 101-111.

Berger-Bachi B. and S. Rohrer. 2002. Factors influencing methicillin resistance in staphylococci. Arch. Microbiol. 178: 165-171. Berglund C., T. Ito, M. Ikeda, X.X. Ma, B. Soderquist and K. Hiramatsu. 2008. Novel type of staphylococcal cassette chromosome mec in a methicillin-resistant Staphylococcus aureus strain isolated in Sweden. Antimicrob. Agents. Chemother. 52: 3512-3516.

Berglund C., T. Ito, X.X. Ma, M. Ikeda, S. Watanabe, B. Soderquist and K. Hiramatsu. 2009. Genetic diversity of methicillin-resistant Staphylococcus aureus carrying type IV SCCmec in Orebro County and the western region of Sweden. J. Antimicrob. Chemother. 63: $32-41$.

Boye K., M.D. Bartels, I.S. Andersen, J.A. Moller and H. Westh. 2007. A new multiplex PCR for easy screening of methicillin-resistant Staphylococcus aureus SCCmec types I-V. Clin. Microbiol. Infect. 13: 725-727.

Boyle-Vavra S., B. Ereshefsky, C.C. Wang and R.S. Daum. 2005. Successful multiresistant community-associated methicillin-resistant Staphylococcus aureus lineage from Taipei, Taiwan, that carries either the novel staphylococcal chromosome cassette mec (SCCmec) type VT or SCCmec type IV. J. Clin. Microbiol. 43: 4719-4730.

Cha H.Y., D.C. Moon, C.H. Choi et al. 2005. Prevalence of the ST239 clone of methicillin-resistant Staphylococcus aureus and differences in antimicrobial susceptibilities of ST239 and ST5 clones identified in a Korean hospital. J. Clin. Microbiol. 43: 3610-3614.

Chambers H.F. and F.R. Deleo. 2009. Waves of resistance: Staphylococcus aureus in the antibiotic era. Nat. Rev. Microbiol. 7: 629-641. Chen L., J.R. Mediavilla, D.C. Oliveira, B.M. Willey, H. de Lencastre and B.N. Kreiswirth. 2009. Multiplex real-time PCR for rapid staphylococcal cassette chromosome mec typing. J. Clin. Microbiol. 47: 3692-3706.

Chlebowicz M.A., K. Nganou, S. Kozytska, J.P. Arends, S. Engelmann, H. Grundmann, K. Ohlsen, J.M. van Dijl and G. Buist. 2010. Recombination between $c c r C$ genes in a type V (5C2\&5) staphylococcal cassette chromosome mec (SCCmec) of Staphylococcus aureus ST398 leads to conversion from methicillin resistance to methicillin susceptibility in vivo. Antimicrob. Agents. Chemother. 54: 783-791.

Chongtrakool P., T. Ito, X.X. Ma et al. 2006. Staphylococcal cassette chromosome mec (SCCmec) typing of methicillin-resistant Staphylococcus aureus strains isolated in 11 Asian countries: a proposal for a new nomenclature for SCCmec elements. Antimicrob. Agents. Chemother. 50: 1001-1012.

Deurenberg R.H., C. Vink, S. Kalenic, A.W. Friedrich, C.A. Bruggeman and E.E. Stobberingh. 2007. The molecular evolution of 
methicillin-resistant Staphylococcus aureus. Clin. Microbiol. Infect. 13: $222-235$.

Deurenberg R.H. and E.E. Stobberingh. 2008. The evolution of Staphylococcus aureus. Infect. Genet. Evol. 8: 747-763.

Francois P., G. Renzi, D. Pittet, M. Bento, D. Lew, S. Harbarth, P. Vaudaux and J. Schrenzel. 2004. A novel multiplex real-time PCR assay for rapid typing of major staphylococcal cassette chromosome mec elements. J. Clin. Microbiol. 42: 3309-3312.

Heusser R., M. Ender, B. Berger-Bachi and N. McCallum. 2007. Mosaic staphylococcal cassette chromosome mec containing two recombinase loci and a new mec complex, B2. Antimicrob. Agents. Chemother. 51: 390-393.

Higuchi W., T. Takano, L.J. Teng. and T. Yamamoto. 2008. Structure and specific detection of staphylococcal cassette chromosome mec type VII. Biochem. Biophys. Res. Commun. 377: 752-756.

Hiramatsu K., L. Cui, M. Kuroda and T. Ito. 2001. The emergence and evolution of methicillin-resistant Staphylococcus aureus. Trends. in microbiology 9: 486-493.

Hiramatsu K., Y. Katayama, H. Yuzawa and T. Ito. 2002. Molecular genetics of methicillin-resistant Staphylococcus aureus. Int. J. Med. Microbiol. 292: 67-74.

Hisata K., K. Kuwahara-Arai, M. Yamanoto et al. 2005. Dissemination of methicillin-resistant staphylococci among healthy Japanese children. J. Clin. Microbiol. 43: 3364-3372.

Ito T., Y. Katayama and K. Hiramatsu. 1999. Cloning and nucleotide sequence determination of the entire mec DNA of pre-methicillin-resistant Staphylococcus aureus N315. Antimicrob. Agents. Chemother. 43: 1449-1458.

Ito T., Y. Katayama, K. Asada, N. Mori, K. Tsutsumimoto, C. Tiensasitorn and K. Hiramatsu. 2001. Structural comparison of three types of staphylococcal cassette chromosome mec integrated in the chromosome in methicillin-resistant Staphylococcus aureus. Antimicrob. Agents. Chemother. 45: 1323-1336.

Ito T., K. Okuma, X.X. Ma, H. Yuzawa and K. Hiramatsu. 2003. Insights on antibiotic resistance of Staphylococcus aureus from its whole genome: genomic island SCC. Drug. Resist. Updat. 6: 41-52. Ito T., X.X. Ma, F. Takeuchi, K. Okuma, H. Yuzawa and K. Hiramatsu. 2004. Novel type V staphylococcal cassette chromosome mec driven by a novel cassette chromosome recombinase, $\mathrm{ccrC}$. Antimicrob. Agents. Chemother. 48: 2637-2651.

IWG-SCC. 2009. Classification of staphylococcal cassette chromosome mec (SCCmec): guidelines for reporting novel SCCmec elements. Antimicrob. Agents. Chemother. 53: 4961-4967.

Katayama Y., T. Ito and K. Hiramatsu. 2000. A new class of genetic element, staphylococcus cassette chromosome mec, encodes methicillin resistance in Staphylococcus aureus. Antimicrob. Agents. Chemother. 44: 1549-1555.

Katayama Y., T. Ito and K. Hiramatsu. 2001. Genetic organization of the chromosome region surrounding mecA in clinical staphylococcal strains: role of IS431-mediated mecI deletion in expression of resistance in mecA-carrying, low-level methicillin-resistant Staphylococcus haemolyticus. Antimicrob. Agents. Chemother. 45: 1955-1963.

Kondo Y., T. Ito, X.X. Ma, S. Watanabe, B.N. Kreiswirth, J. Etienne and K. Hiramatsu. 2007. Combination of multiplex PCRs for staphylococcal cassette chromosome mec type assignment: rapid identification system for $m e c, c c r$, and major differences in junkyard regions. Antimicrob. Agents. Chemother. 51: 264-274.

Kurt K., A. Alderborn, M. Nilsson, B. Strommenger, W. Witte and U. Nubel. 2009. Multiplexed genotyping of methicillin-resistant Staphylococcus aureus isolates by use of padlock probes and tag microarrays. J. Clin. Microbiol. 47: 577-585.

Kwon N.H., K.T. Park, J.S. Moon et al. 2005. Staphylococcal cassette chromosome mec (SCCmec) characterization and molecular analysis for methicillin-resistant Staphylococcus aureus and novel SCCmec subtype IVg isolated from bovine milk in Korea. J. Antimicrob. Chemother. 56: 624-632.

Leski T., D. Oliveira, K. Trzcinski, I.S. Sanches, M. Aires de Sousa, W. Hryniewicz and H. de Lencastre. 1998. Clonal distribution of methicillin-resistant Staphylococcus aureus in Poland. J. Clin. Microbiol. 36: 3532-3539.

Li S., R.L. Skov, X. Han et al. 2011. Novel types of staphylococcal cassette chromosome mec elements identified in CC398 methicillin resistant Staphylococcus aureus strains. Antimicrob. Agents. Chemother.

Ma X.X., T. Ito, C. Tiensasitorn, M. Jamklang, P. Chongtrakool, S. Boyle-Vavra, R.S. Daum and K. Hiramatsu. 2002. Novel type of staphylococcal cassette chromosome mec identified in community-acquired methicillin-resistant Staphylococcus aureus strains. Antimicrob. Agents. Chemother. 46: 1147-1152.

Ma X.X., T. Ito, P. Chongtrakool and K. Hiramatsu. 2006. Predominance of clones carrying Panton-Valentine leukocidin genes among methicillin-resistant Staphylococcus aureus strains isolated in Japanese hospitals from 1979 to 1985. J. Clin. Microbiol. 44: 4515-4527.

Malachowa N. and F.R. DeLeo. 2010. Mobile genetic elements of Staphylococcus aureus. Cell. Mol. Life Sci. 67: 3057-3071.

McCarthy A.J. and J.A. Lindsay. 2010. Genetic variation in Staphylococcus aureus surface and immune evasion genes is lineage associated: implications for vaccine design and host-pathogen interactions. BMC Microbiol. 10: 173.

McClure J.A., J.M. Conly, S. Elsayed and K. Zhang. 2010. Multiplex PCR assay to facilitate identification of the recently described staphylococcal cassette chromosome mec type VIII. Molecular and cellular probes $24: 229-232$.

Milheirico C., D.C. Oliveira and H. de Lencastre. 2007a. Update to the multiplex PCR strategy for assignment of mec element types in Staphylococcus aureus. Antimicrob. Agents. Chemother. 51: 3374-3377.

Milheirico C., D.C. Oliveira and H. de Lencastre. 2007b. Multiplex PCR strategy for subtyping the staphylococcal cassette chromosome mec type IV in methicillin-resistant Staphylococcus aureus: 'SCCmec IV multiplex'. J. Antimicrob. Chemother. 60: 42-48.

Mongkolrattanothai K., S. Boyle, T.V. Murphy and R.S. Daum. 2004. Novel non-mecA-containing staphylococcal chromosomal cassette composite island containing pbp4 and tagF genes in a commensal staphylococcal species: a possible reservoir for antibiotic resistance islands in Staphylococcus aureus. Antimicrob. Agents. Chemother. 48: 1823-1836.

O'Brien F.G., G.W. Coombs, J.C. Pearson, K.J. Christiansen and W.B. Grubb. 2005. Type V staphylococcal cassette chromosome mec in community staphylococci from Australia. Antimicrob. Agents. Chemother. 49: 5129-5132.

Oliveira D.C., A. Tomasz and H. de Lencastre. 2001. The evolution of pandemic clones of methicillin-resistant Staphylococcus aureus: identification of two ancestral genetic backgrounds and the associated mec elements. Microb. Drug. Resist. 7: 349-361.

Oliveira D.C. and H. de Lencastre. 2002. Multiplex PCR strategy for rapid identification of structural types and variants of the mec element in methicillin-resistant Staphylococcus aureus. Antimicrob. Agents. Chemother. 46: 2155-2161.

Oliveira D.C., C. Milheirico and H. de Lencastre. 2006a. Redefining a structural variant of staphylococcal cassette chromosome mec, SCCmec type VI. Antimicrob. Agents. Chemother. 50: 3457-3459.

Oliveira D.C., C. Milheirico, S. Vinga and H. de Lencastre. 2006b. Assessment of allelic variation in the ccrAB locus in methicillinresistant Staphylococcus aureus clones. J. Antimicrob. Chemother. 58: $23-30$. 
Shore A., A.S. Rossney, C.T. Keane, M.C. Enright and D.C. Coleman. 2005. Seven novel variants of the staphylococcal chromosomal cassette mec in methicillin-resistant Staphylococcus aureus isolates from Ireland. Antimicrob. Agents. Chemother. 49: 2070-2083.

Shukla S.K., S.V. Ramaswamy, J. Conradt, M.E. Stemper, R. Reich, K.D. Reed and E.A. Graviss. 2004. Novel polymorphisms in mec genes and a new mec complex type in methicillin-resistant Staphylococcus aureus isolates obtained in rural Wisconsin. Antimicrob. Agents. Chemother. 48: 3080-3085.

Stephens A.J., F. Huygens and P.M. Giffard. 2007. Systematic derivation of marker sets for staphylococcal cassette chromosome mec typing. Antimicrob. Agents. Chemother. 51: 2954-2964.

Takano T., W. Higuchi, T. Otsuka et al. 2008. Novel characteristics of community-acquired methicillin-resistant Staphylococcus aureus strains belonging to multilocus sequence type 59 in Taiwan. Antimicrob. Agents. Chemother. 52: 837-845.

Tsubakishita S., K. Kuwahara-Arai, T. Sasaki and K. Hiramatsu. 2010. Origin and molecular evolution of the determinant of methicillin resistance in staphylococci. Antimicrob. Agents. Chemother 54: 4352-4359.

van der Zee A., M. Heck, M. Sterks, A. Harpal, E. Spalburg, L. Kazobagora and W. Wannet. 2005. Recognition of SCCmec types according to typing pattern determined by multienzyme multiplex PCR-amplified fragment length polymorphism analysis of methicillin-resistant Staphylococcus aureus. J. Clin. Microbiol. 43: 6042-6047.

Wu S.W., H. de Lencastre and A. Tomasz. 2001. Recruitment of the mecA gene homologue of staphylococcus sciuri into a resistance determinant and expression of the resistant phenotype in Staphylococcus aureus. J Bacteriol 183, 2417-2424.

Yang J.A., D.W. Park, J.W. Sohn and M.J. Kim. 2006. Novel PCRrestriction fragment length polymorphism analysis for rapid typing of staphylococcal cassette chromosome mec elements. J. Clin. Microbiol. 44, 236-238.

Zhang K., J.A. McClure, S. Elsayed, T Louie and J.M. Conly. 2005. Novel multiplex PCR assay for characterization and concomitant subtyping of staphylococcal cassette chromosome mec types I to V in methicillin-resistant Staphylococcus aureus. J. Clin. Microbiol. 43: 5026-5033.

Zhang K., J.A. McClure, S. Elsayed and J.M. Conly. 2009. Novel staphylococcal cassette chromosome mec type, tentatively designated type VIII, harboring class A mec and type $4 \mathrm{ccr}$ gene complexes in a Canadian epidemic strain of methicillin-resistant Staphylococcus aureus. Antimicrob. Agents. Chemother. 53: 531-540. 